\title{
Predictions of Quantum Molecular Dynamical Model between incident energy 50 and $1000 \mathrm{MeV} /$ Nucleon
}

\author{
Sanjeev Kumar ${ }^{1, a}$ \\ ${ }^{1}$ Physics and Ballistics Division, State Forensic Science Laboratory, Himachal Pradesh, Shimla Hills, Junga- 171218
}

\begin{abstract}
In the present work, the Quantum Molecular Dynamical (QMD) model is summarized as a useful tool for the incident energy range of 50 to $1000 \mathrm{MeV} /$ nucleon in heavy-ion collisions. The model has reproduced the experimental results of various collaborations such as ALADIN, INDRA, PLASTIC BALL and FOPI upto a high level of accuracy for the phenomena like multifragmentation, collective flow as well as elliptical flow in the above prescribed energy range. The efforts are further in the direction to predict the symmetry energy in the wide incident energy range.
\end{abstract}

\section{Introduction}

The unique theoretical model, which can describe the various phenomena's in the wide range of incident energy for heavy ion collisions, is of great interest these days. In the present era, the theoretical models can be divided into statistical and dynamical models. The dynamical models have the advantage over statistical models to follow the time evolution of any happening from initial conditions till the attainment of equilibrium. In the field of nuclear physics, there are different study regions on the basis of the incident energy of projectile. In the very low incident energy region, the nuclear structure, determination of fusion cross sections, synthesis of super heavy elements takes place $[1,2]$, while from low to intermediate incident energy region, the dominant phenomena's are multifragmentation, collective flow, balance energy, elliptical flow, transition energy, liquid gas phase transition and particles production (pion, kaon, $\Sigma$ ) etc.[3-9]. The high energy region deals with the phenomena of Quark Gluon Plasma (QGP), which is generated by STAR as well as LHC experimental collaborations $[10,11]$. There are different theoretical models developed time to time for the study of these phenomena's. It was found impossible to study above mentioned three energy regions using one theoretical model due to the different physics aspects involved behind them. Out of these, we picked up some of the phenomena's which are taking place in the region above the coulomb barrier upto the intermediate energy region and tried to justify them by using one model known as Quantum Molecular Dynamical (QMD) model.

With the help of this model, we will present here the results for multifragmentation, balance energy as well as elliptical flow from 50 to $1000 \mathrm{MeV} /$ nucleon for heavy-ion collisions. We will also present some results in which it

\footnotetext{
a e-mail: sanjeev1283@gmail.com
}

is shown that Isospin-QMD model, which is modified version of QMD, can be successfully used for the extraction of symmetry energy at low incident energy. Before proceeding towards the results, let us understand briefly the QMD model.

\section{Brief Description of Model}

The QMD model has been successfully applied to extract the information of Nuclear Equation Of State (NEOS) for symmetric nuclear matter. In the model [9], nucleons are represented by the wave packets [12]. The time evolution of the nucleons in the system under the self consistency generated mean field are governed by the well known Hamilton's equations of motion. The Hamilton consists of the kinetic energy and effective interaction potential. The interaction potential is composed of Coulomb, Yukawa, local and momentum dependent interactions, which is written as:

$$
V=V_{\text {Coul }}+V_{\text {Yukawa }}+V_{\text {loc }}+V_{M D I}
$$

where $V_{\text {Coul }}$ and $V_{\text {Yukawa }}$ have the same form as discussed by us and others in the Ref. [9]. The local interaction potential $V_{l o c}$ is originated from the Skyrme energy density function. On the basis of this, the local potential energy density is expanded as:

$$
U_{l o c}=\frac{\alpha}{2} \frac{\rho^{2}}{\rho_{0}}+\frac{\beta}{\gamma+1} \frac{\rho^{\gamma+1}}{\rho_{0}^{\gamma}}
$$

where, $\alpha, \beta$, and $\gamma$ are the parameterized values to specify the particular NEOS. The detailed table is represented in the Refs. [9, 12].

The momentum dependent potential, which is optional in QMD, has the following form[12]:

$$
V_{M D I}=C_{m o m} \ln ^{2}\left[\epsilon(\Delta \vec{p})^{2}+1\right] \frac{\rho}{\rho_{0}} \delta\left(\overrightarrow{r^{\prime}}-\vec{r}\right)
$$


where $C_{\text {mom }}=1.57 \mathrm{MeV}, \epsilon=5 \times 10^{-4} \frac{c^{2}}{{M e V^{2}}^{2}}$ and momentum is given in units of $\mathrm{MeV} / \mathrm{c}$.

With the passage of time, the model is modified for the symmetry energy $\left(E_{S y m}\right)$, which contains potential as well as kinetic energy part. Mathematically, it has been written as follow:

$$
E_{S y m}(\rho)=\frac{C_{s, k}}{2}\left(\frac{\rho}{\rho_{0}}\right)^{2 / 3}+\frac{C_{s, p}}{2}\left(\frac{\rho}{\rho_{0}}\right)^{\gamma_{i}} .
$$

The first term, which is the symmetry kinetic energy, is originated from the Fermi-Dirac distribution. $C_{s, k}=\frac{\hbar^{2}}{3 m}\left(\frac{3 \pi^{2} \rho_{0}}{2}\right)^{2 / 3} \approx 25 \mathrm{MeV}$ is known as the symmetry kinetic energy coefficient. On the other hand, second term is symmetry potential energy, which is adjusted on the basis of calculations from the microscopic or phenomenological many body theory. $C_{s, p}=35.19 \mathrm{MeV}$, is parameterized on the basis of the experimental value of the symmetry energy, is known as symmetry potential energy coefficient. From the Physics point of view, the value of symmetry energy at normal nuclear matter density, using the Bethe-Weizsacker mass formula, should lie between 25 to $32 \mathrm{MeV}$ [13]. Here, in our case, comes out to be 30 $\mathrm{MeV}$, which lies within the range described above.

On the basis of $\gamma_{i}$ value (in Eq. 4), the symmetry energy is divided into two types with $\gamma_{i}=0.5$ and $\gamma_{i}=1.5$, corresponds to soft and hard symmetry energies, respectively.

\section{Results and Discussion}

\subsection{Results of multifragmentation}

In Fig. 1, we display the multiplicity of intermediate mass fragments (IMF's) as a function of the impact parameter for the reaction of ${ }_{79} A u^{197}+{ }_{79} A u^{197}$ at incident energies 100, 400, 600 and $1000 \mathrm{MeV} /$ nucleon. Here soft momentum dependent interaction (SMD) is used with different cross-sections. The legends $\sigma^{55}, \sigma^{40}$ and $\sigma^{\text {Cug }}$ are used for different kind of nucleon-nucleon (NN)cross sections. The $\sigma$ represents cross section and superscript is for the value of cross section used. The Cug is for energy dependent Cugnon cross section[12]. The fragments are made using the minimum spanning tree (MST) method, in which, nucleons are bound if $R_{\text {Clus }}=\left|\overrightarrow{r_{1}}-\overrightarrow{r_{2}}\right| \leq 4 \mathrm{fm}$.

From the figure, the multiplicity of IMF's is maximal at $100 \mathrm{MeV} /$ nucleon for smaller impact parameters, which decreases with the increase in the impact parameter. On the other hand, one sees a rise and fall in the multiplicity of the IMF's at higher incident energies. Due to the low excitation energy, central collisions generate better repulsion and break the colliding nuclei into IMF's, whereas for the peripheral collisions, the size of the fragment is close to the size of the reacting nuclei, therefore, one sees a very few IMF's. In contrary, a rise and fall can be seen at other higher incident energies. For the central collisions, the frequent NN collisions occurring at these energies do not allow any IMF's production. With the increase in incident energy and impact parameter, contribution in IMF's production comes from participant as well as

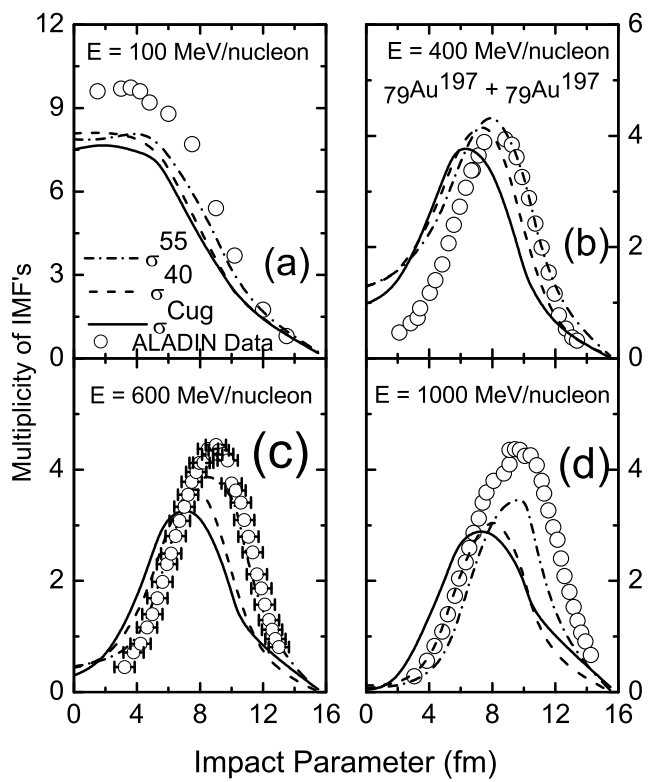

Figure 1. Comparison of average multiplicity of intermediate mass fragments (IMF's) with ALADIN data at incident energies of $100,400 \mathrm{MeV} /$ nucleon (top panel) and 600, 1000 $\mathrm{MeV} /$ nucleon (bottom panel) as a function of impact parameter. The figure is taken from Ref. [21]

spectator matter. Once the contribution of participant matter starts decreasing and size of spectator matter becomes higher than size of IMF's, decrease can be seen in IMF's production.

In all the cases, use of the momentum dependent interaction yields better comparison with ALADIN setup [14] for $\sigma=55 \mathrm{mb}$. This finding is in agreement with the results reported in Refs.[6, 15], where it was found that $\mathrm{NN}$ cross-section has sizable effect on reaction dynamics. Note that in these studies, static equation of state was used.

\subsection{Results of elliptical flow}

The elliptical flow is squeeze out of the particles from the reaction plane. In Fig. 2, we show $v_{2}$ at mid rapidity $\left(|y|=\left|\frac{y_{c . m}}{y_{\text {beam }}}\right| \leq 0.1\right)$ for $Z \leq 2$ (left panel) and for protons (right panel) as a function of the incident energy for the reaction of ${ }_{79} A u^{197}+{ }_{79} A u^{197}$. The theoretical results are compared with the experimental data extracted by INDRA, FOPI and PLASTIC BALL collaborations[16, 17]. With the increase in the incident energy, elliptical flow $v_{2}$ changes from positive to negative values exhibiting a transition from the in-plane to out-of-plane emission of nucleons. This is because of the fact that the mean field, which contributes to the formation of a rotating compound system, becomes less important and the collective expansion process based on the NN scattering starts to be predominant. This competition between the mean field and NN collisions depends strongly on the effective interactions that leads to the different transition energy due to different equations of state. Due to the repulsive nature of the momentum dependent interactions, 


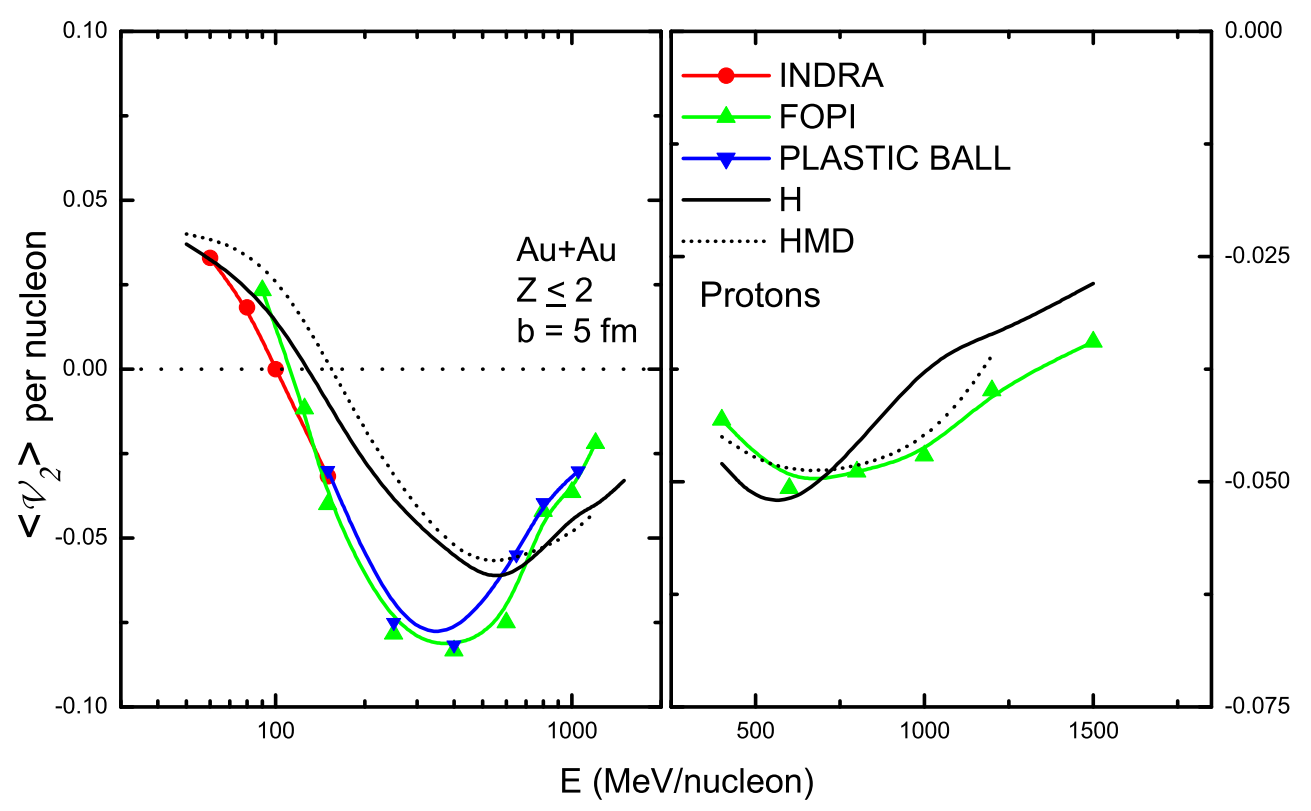

Figure 2. The variation of the elliptical flow, summed over entire transverse momentum, with beam energy at $|y|=\left|\frac{y_{c . m}}{y_{\text {beam }}}\right| \leq 0.1$ for ${ }_{79} A u^{197}+{ }_{79} A u^{197}$ reaction. Here comparison is shown with experimental findings of INDRA, FOPI and PLASTIC BALL Collaborations[16, 17]. The figure is taken from Ref. [25]

which leads to the suppression of binary collisions, less squeeze-out is observed in the presence of momentum dependent interactions (HMD) compared to static one $(\mathrm{H})$. The maximal negative value of $v_{2}$ is obtained around $E=500 \mathrm{MeV} /$ nucleon with hard $(\mathrm{H})$ and hard momentum dependent (HMD) equations of state. This out-of-plane emission decreases again towards the higher incident energies. This happens due to faster movement of the spectator matter after $v_{2}$ reaches the maximal negative value [17]. This trend is in agreement with experimental findings. A close agreement with data is obtained in the presence of hard equation of state for $Z \leq 2$ particles, while, in the presence of momentum dependent interactions for protons. Similar results and trends have also been reported by Zhang et.al. in their recent communication [18].

\subsection{Results of Directed flow: Balance energy}

The balance energy is the energy at which directed flow becomes zero. The directed flow is the measure of movement of particles in the direction of impact parameter. In Fig. 3, we display the energy of vanishing flow or balance $\operatorname{energy}\left(E_{b a l}\right)$ as a function of composite mass of system that ranges from ${ }_{18} A r^{40}+{ }_{21} S c^{45}$ to ${ }_{79} A u^{197}+{ }_{79} A u^{197}$. The balance energy has been shown for the experimental data (open stars), 40mb-QMD (crossed triangle) and IQMD+0.9 $\sigma_{N N}$ (solid squares). All the curves are fitted with power law of the form $C\left(A_{T O T}\right)^{\tau}$. The difference is observed in the $\tau$ values obtained by different theoretical models[19]. In the BUU model, the $\tau$ value has been reported between $-0.28 \leq \tau^{\text {th }} \leq-0.32$. In modified work with BUU model[20], the $\tau^{\text {th }}$ value is found to be

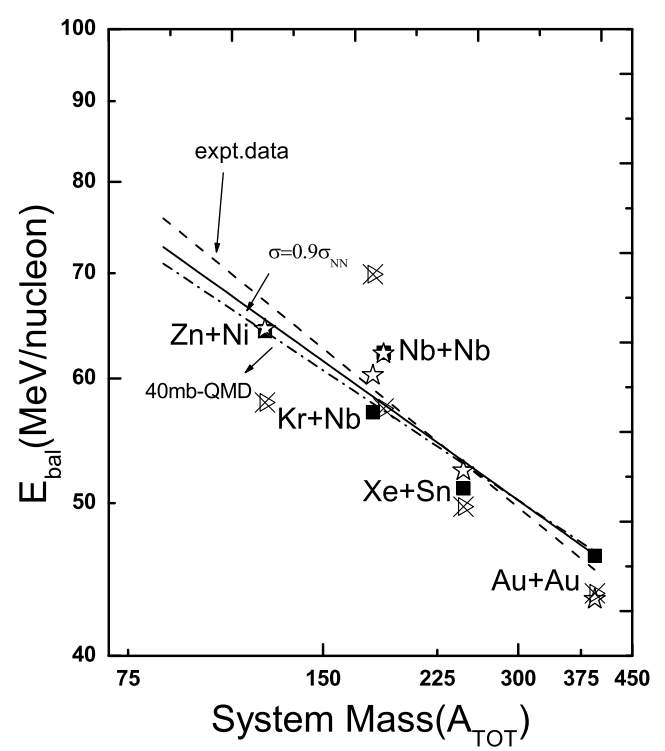

Figure 3. Balance energy as a function of combined mass of the system. The experimental points are represented with stars, $40 \mathrm{mb}-\mathrm{QMD}$ with crossed triangle and present with solid square. The figure is taken from Ref. [23]

$-0.41 \pm 0.03$. The present calculation depicts the $\tau$ value $(-0.29 \pm 0.06)$, which is close to the experimental $\tau$ value $(-0.33 \pm 0.06)$ as compared to $40 \mathrm{mb}-\mathrm{QMD}$ calculation having $\tau$ value $(-0.27 \pm 0.17)$. In other words, the present IQMD model with a stiff equation of state along with $\sigma=0.9 \sigma_{N N}$ can explain the data much better than any other theoretical calculations for heavier nuclei. The lighter nuclei, when checked out, demand for an enhanced cross-sections $[19,21]$ along with momentum dependent 


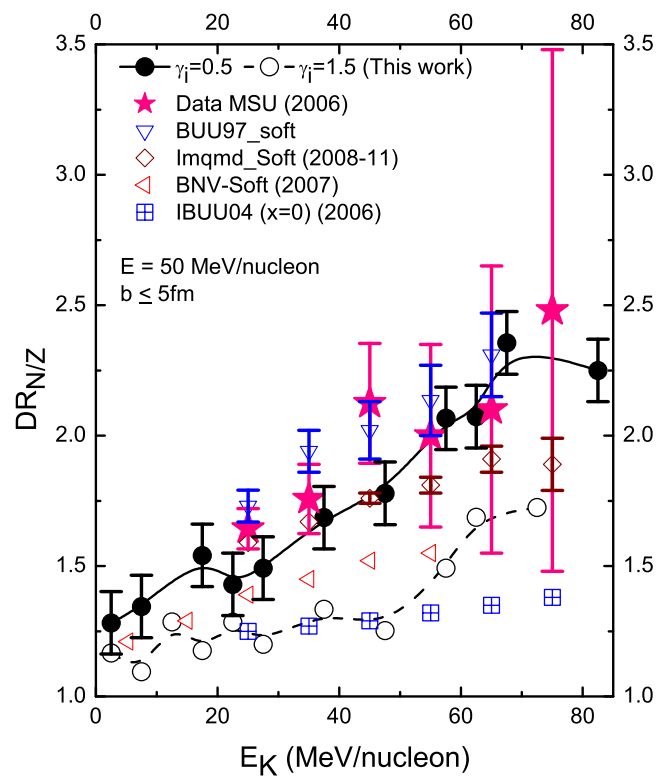

Figure 4. The comparison of free neutrons to protons double ratio at $\mathrm{E}=50 \mathrm{MeV} /$ nucleon and $b \leq 5$ with the MSU/NSCL data, BUU97, IBUU04, BNV, and ImQMD simulations. The figure is taken from Ref. [27]

interactions [22]. Our calculations about the strength of reduced $\mathrm{NN}$ cross-section is in agreement with earlier calculation, where disappearance of transverse in-plane flow [24] as well as elliptical flow is parameterized with experimental data [26].

\subsection{Predictions of symmetry energy}

In order to study the symmetry energy, the double $n / p$ ratio of $S n^{124}+S n^{124}$ to $S n^{112}+S n^{112}$ is calculated. In Fig. 4, all the possible results of double ratio available in the literature, are compared with the results from IQMD model. Let us start with very first comparison of the BUU97 [28]. The results were very close to the experimental one, but, the reaction conditions were different. Firstly, in the BUU97 calculations, the incident energy was $40 \mathrm{MeV} /$ nucleon, not $50 \mathrm{MeV} /$ nucleon, just like the experimental one. Secondly, data set is only for the transverse emission, while in the BUU97 calculations, the nucleons used are emitted in all the directions. Move one step ahead to the IBUU04 results [29], where the symmetry energy is introduced with the help of momentum dependent interactions, the results are very far from the experimental data. The same is true for the BNV calculations performed by the Catania group in 2007 [30]. The most closeness between the data and the calculation is observed by the ImQMD model in 2009 [31]. They found that the results with $\gamma_{i}=0.75$ are best fit with the experimental data for impact parameter $b \leq 2 \mathrm{fm}$. In the present study, we have performed simulations for $b \leq 5 \mathrm{fm}$ with the soft and stiff symmetry energy and found that the results obtained with $\gamma_{i}=0.5$ of IQMD model are very close to experimental data.

\section{Conclusion}

The QMD model is a successful model in the low to intermediate incident energy range to explain many phenomena like multifragmentation, elliptical flow, balance energy and extraction of symmetry energy. The findings for the symmetric nuclear matter reveals the soft nature of the hadronic matter. On the other hand, the findings of symmetry energy are indicating the soft symmetry energy nature of asymmetric nuclear matter. The extraction of symmetry energy at higher incident energy is a question of hot debate these days.

\section{References}

[1] J. Dong et al., Phys. Rev. Lett. 107, 012501 (2011).

[2] C. Rizzo et al., Phys. Rev. C 83, 014604 (2011).

[3] C. Hartnack et al., Phys. Rev. Lett. 96, 012302 (2006).

[4] C. Hartnack et al., Phys. Rep. 510, 119 (2012).

[5] Y. K. Vermani et al., Phys. Rev. C 79, 064613 (2009).

[6] R. K. Puri et al., J. Comput. Phys. 162, 245 (2000).

[7] A. D. Sood et al., Phys. Rev. C 73, 067602 (2006).

[8] A. D. Sood et al., Phys. Rev. C 70, 034611 (2004).

[9] C. Hartnack et al., Eur. Phys. J. A1, 151 (1998).

[10] M. D. Toro et al., Phys. Rev. C 83, 014911 (2011); F. M. Liu and S. X. Liu, Phys. Rev. C 89, 034906 (2014).

[11] J. L. Nagle et al., New J. Phys. 13, 075004 (2011).

[12] J. Aichelin, Phys. Rep. 202, 233 (1991).

[13] C. Samanta and S. Adhikari, Phys. Rev. C 65, 037301 (2002).

[14] A. Schüttauf et al., Nucl. Phys. A607, 457 (1996).

[15] Y. K. Vermani et al., Eur. Phys. Lett. 85, 62001 (2009).

[16] J. Lukasik et al., Phys. Lett. B608, 223 (2005).

[17] A. Andronic et al., Nucl. Phys. A679, 765 (2001); Phys. Lett. B612, 173 (2005).

[18] Y. Zhang and Z. Li, Phys. Rev. C 74, 014602 (2006).

[19] A. D Sood et al., Phys. Rev. C 69, 054612 (2004); Phys. Rev. C 79, 064618 (2009).

[20] D. J. Magestro et al., Phys. Rev. C 61, 021602(R) (2000).

[21] S. Kumar et al., Pramana J. of Physics 74, 731 (2010).

[22] Y. K. Vermani et al., J. Phys. G 36, 105103 (2009); J. Phys. G 37, 015105 (2010); S. Kumar et al., Phys. Rev. C 78064602 (2008).

[23] S. Kumar et al., Phys. Rev. C 82, 024610 (2010).

[24] S. Gautam et al., J. phys. G 37, 085102 (2010).

[25] S. Kumar et al., Phys. Rev. C 81, 014611 (2010).

[26] Y. M. Zheng, Phys. Rev. Lett. 83, 2534 (1999)

[27] S. Kumar et al., Phys. Rev. C 84, 044620 (2011).

[28] B. A. Li et al., Phys. Rev. Lett. 78, 1644 (1997).

[29] B. A. Li et al., Phys. Lett. B 634, 378 (2006).

[30] M. Colonna et al., Phys. Rev. C 78, 064618 (2008).

[31] Y. Zhang et al., Phys. Lett. B 664, 145 (2008); M. B. Tsang et al., Phys. Rev. Lett. 102, 122701 (2009). 